\section{Diagnosis of meconium aspiration by spectrophotometric analysis of urine}

We noticed that newborn infants with signs of respiratory distress due to meconium aspiration frequently pass dark urine soon after birth. In the most striking cases the urine has a chestnut-brown colour. In searching for an explanation for this the possibility arose that a water-soluble substance derived from meconium might be absorbed through the lungs and excreted in the urine. To investigate this we compared spectrophotometric measurements on aqueous extracts of meconium with measurements of urine from infants with and without clinical signs of meconium aspiration.

\section{Material and methods}

Urine passed within 24 hours of delivery by 15 normal infants and by 12 infants with evidence of meconium aspiration was collected in glass containers. Attention was given to avoiding contamination with meconium during collection. The urine samples were immediately frozen at $-20^{\circ} \mathrm{C}$ and protected from exposure to light. Some samples of meconium were preserved under the same conditions, and later diluted in aqueous solutions for spectrophotometric studies.

Diagnosis of meconium aspiration was made only when the following signs were all present: evidence of fetal distress; passage of meconium-stained amniotic fluid before delivery; an Apgar score of 5 or . less within the first 5 minutes of life; respiratory distress with signs of airway obstruction; the presence of meconium or meconium-stained liquid in the trachea; and a characteristic $x$-ray appearance as described by Gooding and Gregory (1971).

After the urine samples were thawed, we compared the colour of these samples with an arbitrary scale ranging from pale yellow to dark brown. The samples were then analysed by spectrophotometry using a DB-GT Beckman apparatus. The optical density of the samples was measured in a $1 \mathrm{~cm}$ light path cuvette against distilled water, and expressed on a linear scale (Fig. 1A). The absorption band at about $405 \mathrm{~nm}$ observed on the spectra of meconiumcontaining urine was estimated as a urinary meconial index (UMI) as shown in Fig. $1 B$. We also applied the spectrophotometric method to the detection of meconium pigment in samples of amniotic fluid obtained by amniocentesis late in pregnancy.

\section{Results}

As shown in Fig. $1 A$, the absorption spectrum for a solution of meconium in water contains a peak at $405 \mathrm{~nm}$. A peak in the same position can be seen in urine from infants with meconium inhalation (Fig. $1 B)$, and in samples of meconium-stained amniotic fluid (Fig. 1C) but not in normal urine (Fig. 1D). We conclude that the peak at $405 \mathrm{~nm}$ in urine is due to the excretion of water-soluble pigments derived from meconium. In 5 infants with meconium aspiration from whom serial samples of urine were collected, the meconial peak decreased rapidly, usually disappearing within 24 hours.

Fig. 2 shows values of UMI in the two groups of patients. There is a clear separation between them; none of the normal infants had an index above 1, whereas all the affected infants had a higher value.

Although darkly-stained urines always gave a high value for the UMI, and clear urines frequently gave a normal value, intermediate colours correlated poorly with the spectrophotometric findings.

\section{Discussion}

Simple inspection of urine specimens allows confirmation of a diagnosis of meconium aspiration when it is darklystained, or rejection of this diagnosis when it is obviously clear. When the colour of the urine samples is not so clearly different, only a spectrophotometric study can make the distinction between normal and abnormal urine.

The exact nature of the substance present in the meconial urines is as yet unspecified. Studies of the pigment composition of meconium have shown that several products resulting from the catabolism of haemoglobin could absorb light at about $405 \mathrm{~nm}$, such as mesobilifuchsins, porphyrins, some derivatives of bilirubin (Rodriguez Garay et al., 1964).

The value of UMI does not seem to correlate with the gravity of the pulmonary disorder. The test must therefore be regarded for the present as qualitative. This is not surprising, because the severity of the pulmonary disorders caused by meconium aspiration depends upon so many inter-related factors such as the intensity and duration of fetal distress, the amount and consistency of the meconium inhaled, the efficiency of immediate endotracheal suction in the delivery room (Gregory et al., 1974), and the thickness of the aspirated fluid.

The differential diagnosis of respiratory disorders in newborn infants presents many difficulties. In the early stages it is often impossible on clinical or 

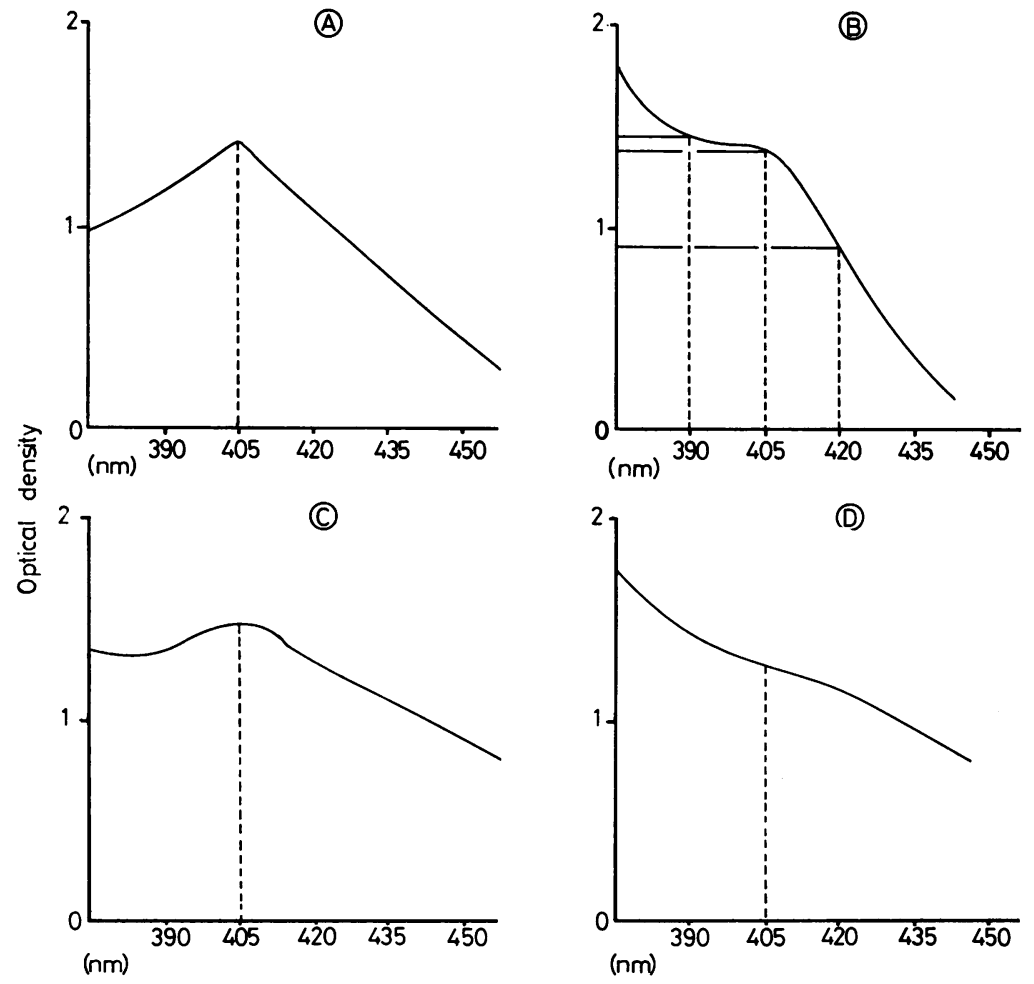

Fig. 1 A: Spectrum of an aqueous extract of meconium; B: spectrum of the first urine passed by a newborn with meconium inhalation. Urinary meconial index, UMI $=\left[O D_{405}-\left(\frac{O D_{390}+O D_{420}}{2}\right)\right] \times 50$;

$C$ : spectrum of a meconium-stained amniotic fluid, and D: of the first urine passed by a normal infant.

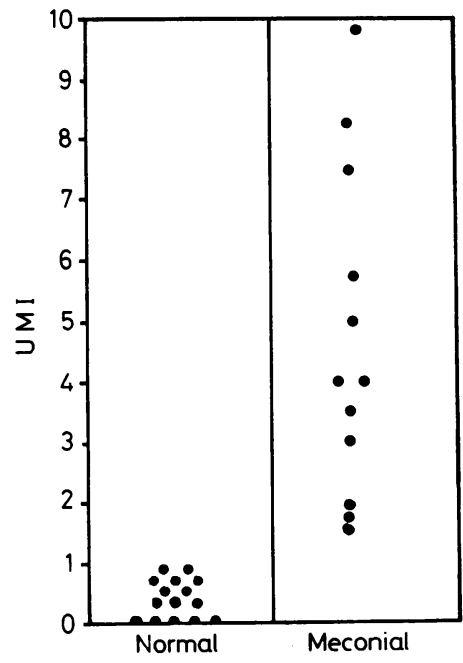

Fig. 2 Urinarymeconial index(UMI) in 15 normal newborn infants and in 12 newborn infants with meconium inhalation. radiological grounds to distinguish between hyaline membrane disease, lung oedema, congenital pneumonia, transient tachypnoea, and aspiration syndromes, and indeed several of these conditions may coexist. The present method of detecting meconium pigment in infants' urine may help to disentangle these complicated situations by identifying more clearly one element, meconium inhalation.

\section{Summary}

The first urine passed by a newborn infant who has inhaled meconium or meconium-stained amniotic fluid often contains a dark-coloured water-soluble substance, recognisable spectrophotometrically by an absorption band at about $405 \mathrm{~nm}$. Spectrophotometric analysis of the first urine can in this way be used to diagnose preceding meconium aspiration.

This work was supported by the Institut National de la Santé et de la Recherche Médicale (INSERM). 


\section{References}

Gooding, C. A., and Gregory, G. A. (1971). Roentgenographic analysis of meconium aspiration of the newborn. Radiology, 100, 131-135.

Gregory, G. A., Gooding, C. A., Phibbs, R. H., and Tooley, W. H. (1974). Meconium aspiration in infants: a prospective study. Journal of Pediatrics, 85, 848-852.

Rodriguez Garay, E., Lozzio, B., O’Donnell, J. C., Toccalino, H., and Emiliani, R. (1964). Les pigments biliaires du méconium humain. Revue Internationale d'Hépatologie, 14, 323-334.

Michel Dehan, Jeanne Francoual, and Albert LINDENBAUM

Service de Réanimation Néonatale and Laboratoire de Biochimie, Hôpital Antoine Béclère 92140 Clamart, France.

Correspondence to Dr M. Dehan.

\section{Establishing demand feeding in hospital}

It has been suggested that infants should be fed when they wake up and cry for a feed, 'demand feeding', rather than according to any rigid schedule, "clock feeding' (Woody and Woody, 1966; Mac Keith and Wood, 1971). However, many hospitals, including our own, have in the past continued to feed babies according to a rigid schedule in the belief that demand feeding would be incompatible with ward efficiency. In the summer of 1975 it was decided to introduce demand-feeding for both breast- and bottlefed babies throughout the hospital for a trial period. At the time of this study $65 \%$ of all mothers leaving the hospital were breast feeding their babies.

\section{Methods}

Demand feeding instructions were sent out to all the midwifery staff in the last week of July 1975 , to be introduced on August 1, and was defined as follows. The mother was to be encouraged to pick up her baby whenever she felt that he was hungry and to offer him a feed. She was also to be encouraged to have her baby at her bedside at night. If a mother chose to have her baby looked after in the ward nursery (each 4-bedded maternity ward has a nursery for 4 cots adjacent), instructions were given for the baby to be brought to the mother if he awoke. In addition, the practice of giving formula milk feeds to breast-fed babies, either as complements or supplements, was to be generally discouraged. Where additional feeds were thought to be indicated, water was to be offered, except in the case of small-fordates infants considered to be at risk of developing hypoglycaemia, who were to be allowed formula feeds in the early neonatal period.
For the last 10 days in July all mothers, except those in the isolation unit, those whose babies were in the special care baby unit, and those whose babies were small-for-dates, were asked to complete detailed feed charts. The mothers recorded the time of each feed, and the number and nature of supplementary and complementary feeds. The charts were completed throughout the mothers' stay in hospital. Because we were mainly interested in the effects of demand feeding on breast-fed babies, only the charts of breast-fed singleton babies were analysed. There were 46 such charts of which 4 were rejected because incomplete.

During August all mothers (except those excluded as above) completed feed charts during their stay in hospital. Charts completed during the second half of August, when demand feeding had become well established, were analysed for comparison with the July charts. Of 57 breast feeding charts of singleton babies in this period, 2 were rejected because incomplete. The distribution of the remaining 55 babies between the four maternity wards showed an excess of babies from one ward compared with the clock-fed group. In order to obtain a sample with the same distribution between the wards as the initial clock-fed sample, a group of 8 was randomly selected from the 20 on the ward for inclusion in the analysis.

In addition, information was collected on the infants' weight gain and the occurrence and severity of jaundice among the two groups of babies. At the end of August mothers and nursing staff were asked to record their impressions of the new ward routine.

\section{Results}

Parity, birthweight, and gestational age. There was no difference in parity between the two groups of mothers. The birthweights of the babies in the two groups were similar (clock-fed: mean $3455 \pm$ SD 458 $\mathrm{g}$; demand-fed: mean $3535 \pm 466 \mathrm{~g}$ ), as were gestational ages (39.8 \pm 1.4 weeks; and $40 \cdot 1 \pm 0.8$ weeks).

Time intervals between feeds. The pattern of feeding was analysed according to the infants' postnatal age in days. In each case the number of days was calculated in 24-hour periods from the time of birth. The time intervals between feeds on the first and second days within each group were similar, as were the intervals on the 5th, 6th, and 7th days. The results are therefore presented in terms of these two groups of days, representing the beginning and end of the first week after birth.

During the first and second days after birth there was a significant difference between the demand-fed and clock-fed groups in the distribution of time intervals between feeds $\left(P<0.001\right.$ using the $x^{2}$ test $)$. 